\title{
Evaluation of seeds quality in the fruits of cherry varieties (Cerasus vulgaris L.) in the process of embryos cultivation in vitro
}

\author{
Natalia Kovalenko*, Svetlana Gladkih \\ Krymsk EBS VIR Branch, Vavilov str., 12 Krymsk, 353384, Russia
}

\begin{abstract}
Annotation. The objective reasons for the low yield of breeding material are shown. They are associated with the timing of the fruits selection, affecting the formation of the embryos and cotyledons, as well as their infection and damage by seed eater (Apion Pomonae Fabricius). The paper presents a general characteristics of the seeds quality of cherry varieties and the stages of development of embryos and cotyledons depending on the period of pollination. The presence of damage to the seed by seed eaters at an early stage was revealed, and then the complete destruction of the nucleus by its larvae. The assessment of the seeds quality in the fruits of cherry varieties $(C$. vulgaris $\mathrm{L}$.) was carried out to reveal the suitability their embryos cultivation in vitro for breeding programs of new varieties development. The presence of three waves of fruit dropping, inherent in cherry varieties after blossoming is clarified. When assessing the quality of the seed of the varieties introduced into in vitro culture, it is necessary to pay attention to the timing of the "harvest" of fruits and their infection, the formation of the embryos and cotyledons, as well as the presence or absence of clutches of seed eaters (damage by seed eaters).
\end{abstract}

\section{Introduction}

The breeding process to create the varieties more productive, early-ripening and resistant to major harmful diseases than the existing varieties of sweet cherries and cherries presented in the State Register continues in the scientific institutions of the Russian Federation, including at the Krymsk Experimental Breeding Station of the VIR branch using the biotechnological methods [1].

Cultivation of hybrid embryos of cherry [2, 3] and sweet cherry $[1,4,5]$ in vitro makes it possible to grow them and accelerate the appearance of new forms. The modern biotechnology in this area is based on knowledge of nutrient media proposed earlier and is supplemented by new knowledge about the composition of the artificial nutrient media used $[4,5,6]$, and the effect of disinfecting preparations the embryos infection $[1,7,8]$. Inspite on success in solving of these problems by biotechnologists, the yield of breeding material is not high, and in order to optimize seed germination in Prunus L. species, the various their

* Corresponding author: kross67@mail.ru 
treatments are proposed, for example, preliminary exposure to hydrogen peroxide or gibberellic acid in combination with stratification [9].

In experiments in vitro culture, it is undoubtedly very important to take into account the qualitative composition of seeds in the fruits. In such studies, very little attention was paid to this, and the results of the experiments were evaluated almost exclusively by the number of seedlings obtained, which number depends on many factors affecting the fruits formation. These factors include also the chemical substances, the processing of which increases in the yield capacity [10]. The genotype - environment interaction also plays an important role in the period of pollination [11].

For the successful cultivation of embryos in vitro, a full fertilization, formation and infection of the seed are of great importance. It has already been determined that the place of infection in Prunus L. species is mainly the epidermis for viruses (for example, Scharka) and the aphids are the active vectors [12]. When embryos are introduced into in vitro culture, self-incompatibility assessment has recently been used [13]. The timing of its isolation in the early stages of development is also associated with this. Up to 30 days after pollination, it is very rarely possible to obtain the seedlings, in comparison with later ones, since after 50-55 days the embryo differentiates into a bud, root and cotyledon.

Fertilization plays an important role in the development of the embryo, after that there is an enhanced development of the endosperm, the embryo and an increase in the size of the ovary itself. Fruit growers are well aware that not all of these ovaries will give the ripen fruits, since they fall off at different stages of development, and it must be taken into account by the biotechnologist.

The reasons that cause the ovary falling off have been found out by many researchers in the last century. In particular, for sweet cherries and cherries, Dorothy Bradbury (1925) has been pointed out in her work that the fall of their fruits occurs in three waves, which are sharply distinguishable: the fruits falling in the first two waves are easily distinguishable in many days before they fall off, from those remaining on the tree branch until the next fall, or until they are fully ripening. Based on accurate anatomical studies, D. Bradbury concluded:

- the first ovary abscission is caused not by a lack of pollination or too weak growth of the pollen tube, but by the lack of fertilization; at the same time, $82-99 \%$ of the ovaries of the first falling off had the pollen tubes, and in 76-90\% of all embryos studied, the pollen tubes reached the ovule; the reason for the prolapse in this case is the degeneration of the egg cell and as a result of this, the act of fertilization does not occur;

- the second abscission is caused mainly by the abortion of the embryo sac or the death of the embryo (41\% had embryos, which indicates fertilization); under the influence of insufficient nutritional conditions, the embryos stopped in their development;

- the third wave of fruit abscission is associated with aborted embryos and endosperm (as well as in the second), but their development stops later, which indicates a struggle for nutrition; in this case, the reason for the falling off of the ovaries is mainly the conditions of insufficient nutrition of the fruits.

According to the research of D. Bradbury, all ovaries of the third period abscission are normally fertilized and contain embryos in various degrees of development. These results were confirmed by A.J. Farley (1927), H. Kamlah (1928) and M.B. Crane (1923).

The study of the mechanisms of ovary shedding, and their quality, development and growth of embryos allows us to solve the arising problems of creating a selection fund for cherry ordinary and ducks using in vitro. This area of biotechnology has not been sufficiently researched and developed. In this regard, a conformity need is to study the biology of the ovary development and the quality of seeds introduced into culture. 


\section{Materials and methods}

The work was carried out at the VIR Collections of Plant Genetic Resources within the framework of the VIR State Assignment (budget project No. 0662-2019-0004).

Studies on the evaluation of seeds in the fruits of cherry varieties (Cerasus vulgaris L.) to optimize the timing of the isolation of embryos during in vitro cultivation were carried out in the laboratory of biotechnology and biochemistry of the Krymsk EBS branch of VIR (2018-2020).

The material was 12 collection cherry varieties of various origins. These included the varieties of ordinary cherries (Turgenevka, Lyubskaya, Shishevskaya, Shahrazada) and dukes (Igrushka, Chudo Vishnya, Erdi Krupnoplodnaya, Krasavitsa Ribokura, Prevoskhodnaya Venyaminova, Yuzhnaya Krasavitsa, Malyshka, Effectivnaya).

The time of mass blossoming was visually determined, the date of pollination and ovary shedding was fixed. A Digital model 1000x microscope was used to study the state of the fruit seed.

Sterilization against the surface saprophytic microflora of fruits, stones and seeds was carried out according to the scheme worked out in the laboratory: washing in a soapy solution $=>$ rinsing fruits with water $=>$ separating seeds and processing them with a chlorinecontaining aqueous solution of Deo Chlorine $=>$ scarification and disinfection of the core in a two-phase method: a chlorine-containing aqueous solution Deo Chlorine and Hydrogen Peroxide.

The embryos treated were planted in the sterile tubes $21.0 \times 140 \mathrm{~mm}$ in size with agar culture medium based on Murashige and Skoog (1962) with additions and modifications [1]. The results of the experiments were fixed in observation logs, as well as with the help of a camera.

\section{Results and discussion}

Phenological observations of collection cherry varieties under the climatic conditions of the Foothill zone of the Krasnodar Territory, where the Krymsk EBS is located, have confirmed an information from literary sources about three waves of fruit abscission after blossoming. The unfertilized or partially fertilized seeds introduced into the culture when planted on nutrient media in vitro did not give the seedlings in our experiments.

The fruits of the first abscission are not of interest to the breeder, since the act of fertilization does not occur due to the lack of growth of the pollen tube or, when the pollen reaches the ovule with a degenerative egg cell. Such fruits fell off along with the calyx almost immediately after blossoming and were not included in the experiments, as well as the fruits from the second fall off, which embryos turned brown and then dried out.

When examining a section of a cherry ovary under a microscope, we observed the developing cotyledons and an embryo (Fig. 1a) or an incompletely differentiated fertilized embryo (aborted) (Fig. 1b). 


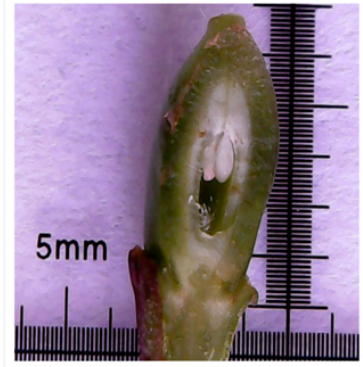

A

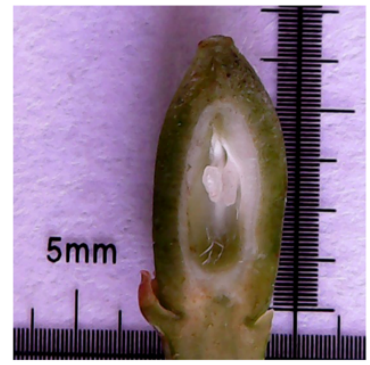

B

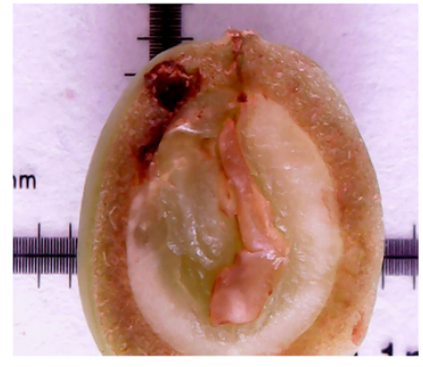

C

Fig. 1. Ovary of Shishevskaya cherry after fertilization (a, b - on 12 day; $\mathrm{c}-$ on 19 day)

$$
\text { A - normal development } \quad \text { B - abortion of the embryo }
$$

C - fruit affected by vermins and diseases

The observations have shown that the abortion of the embryo took place in the subsequent stages of its development after fertilization and most often due to a lack of fruit nutrition in their struggle for existence. If the more fruits were set, the more ones fell off, which is associated with cessation in the fruit development and their rejection.

For cherry, a "turning point" in the shedding of ovaries, associated with defective egg mechanism or incompatibility with pollen, occurs in 2-3 weeks after pollination (14-20 days). Therefore, on the 19-23 day of fruit growth, their embryos are ready for in vitro development, but this period coincides with the greatest opportunities for vermins intervention (Fig. 1c, 2a). Thus, in our experiments during the scarification of the stones, the Apion Pomonae clutches and larvaes were found inside the kernel (Fig. 2a, 2b).

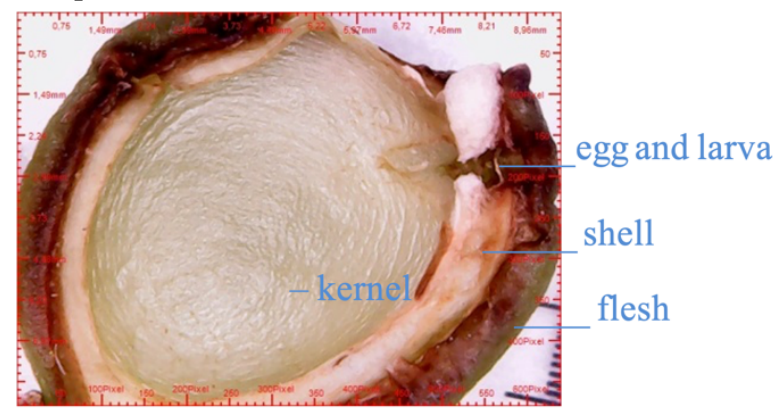

a

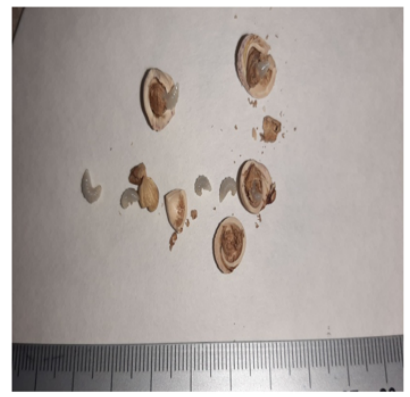

6

Fig. 2. The cherry fruit and kernel infected by Apion Pomonae

We classified as apionids the numerous species of hymenoptera, belonging to the families of Eurytomidae and Callimomidae of $2.4 \mathrm{~mm}$ long, and secondarily herbivorous. The presence of seed eaters in the cherry fruits is the reason for decrease in the yield of seedlings when using in vitro culture at an early and later stages of embryo development. The Apion Pomonae clutches are died during sterilization, but a damaged embryo (or whole seed) remains.

If on the 14-20th day after fertilization the cotyledons are infected with the seed-eater, which can be seen only with magnifying, then on the 50-60 day the fruits infected with the seed-eater don't have the cotyledons and the embryo, and they are completely eaten by the larvaes, that we observed during scarification (Fig. 2b).

When the fruits are taken in their incomplete maturity for introduction into the embryos culture, there is a possibility of "getting the fruits into experiments" from the third wave of abscission, which is associated with abortion of both the endosperm and the embryo due to 
the struggle for nutrition or, like in dukes, due to postgamous incompatibility. The reason for the poor quality of the seeds may be the discrepancy in the time between development of the endosperm and the embryo itself.

When cherry embryos are cultivated in vitro, the seedlings developed from them, both normally formed and with defects (Fig. 3).

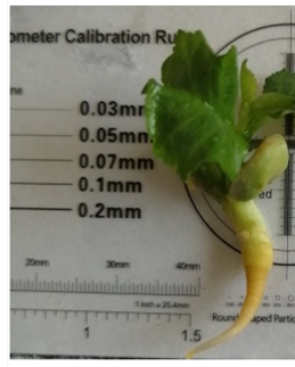

a

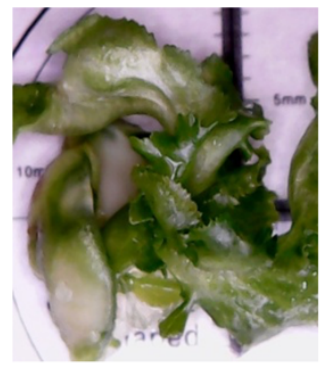

b

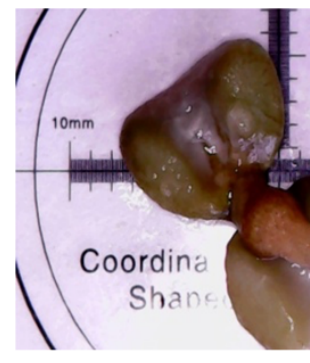

$\mathrm{c}$

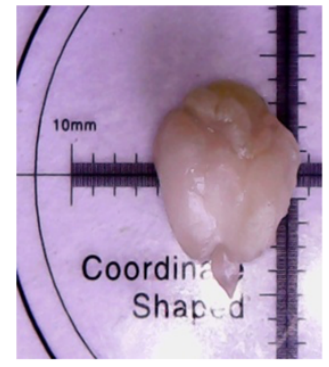

d

Fig. 3. The development of embryos during cultivation in vitro on days 18-20

Figure 3 shows the various options for the embryos development in vitro culture: 1) a well-developed cherry seedling, with stem and root (fig. 3a), which can later be transferred ex vitro and planted in vivo; 2 ) abnormal development of the embryo without root and stem (fig. 3b, 3c), these embryos are micropropagated by introducing tissue (parts of organs) into the culture [1]. In this case, it is possible to use the knowledge of foreign scientists about the introduction of sweet cherry (Ksenia) and cherry (Vasilisa Prekrasnaya) [14] as well as the innovative approaches to the cultivation of sweet cherry plants [15]. With a weak development of the cotyledons, the embryo is incapable of normal germination and can to develop only on an artificial nutrient medium, and in the case of its isolation from the cotyledons. The albino seedlings are rejected (fig. 3d). The percentage of "non-arising embryos" also includes the seeds that have stopped the development, the seeds with internal infection and cotyledons developed without an embryo.

\section{Conclusion}

According to the results of observations during the period of the first and second shedding of cherry fruits, there is a high probability that fertilization did not occur and the embryo died in the maternal tissues. The embryos not enough developed can also be in the fruits during the third period of their abscission, when there is a competition for nutrients between them, which corresponds to 19-23 days from fertilization. The percentage of seedlings yield when using a culture of embryos is very strongly influenced by their infection with seed-eaters, when not only the cotyledons, but also the embryo are affected, including internal infection, in general, all this leads to a very low efficiency of hybridization.

In order to avoid distortion of the true picture of the use of the culture of cherry embryos in vitro, it should be taken into account that the timing of the "harvest" of fruits for isolation of the seed is closely related to the timing of fruits abscission. And if the fruits from the first wave of abscission, practically after blossoming are not included in the breeding process, then the fruits used from the second wave of abscission (14-20 days after pollination), highly likely do not have an embryo and, therefore, there is no possibility to get the seedlings.

The fruits of later ripening may contain the embryos with various degree of their formation associated with a lack of nutrition, underdevelopment of the cotyledons, infection and damage by Apion Pomanae Fabricius, which directly negatively affect the yield of breeding material. 
Under the field conditions, the seeds with the above noted qualities will give the only single seedlings, but the number of seedlings significantly increases when using the biotechnological methods, such as isolating the embryo from the cotyledons underdeveloped, and the using a culture of organs and tissues with abnormal embryo development (without a root or without a shoot). In this case, the cherry seedlings develop from normally formed embryos in the year of crossing and, when using micropropagation, if it necessary, they can be cloned. All this significantly increases in the yield of breeding material and accelerates the process of obtaining the new cherry varieties.

\section{References}

1. N. N. Kovalenko, S. V. Gladkih, Vavilov Journal of Genetics and Breeding, 23(6), 765-771 (2019). http://doi.org/10.18699/VJ19.550

2. T. V. Plaksina, D. S. Kulhanova, E. Y. Matkova, Achievements of Science and Technology of AIC, 7, 17-19 (2013). https://cyberleninka.ru/article/n/kulturaizolirovannyh-zarodyshey-in-vitro-v-prakticheskoy-selektsii-vishni

3. D. Dorić, V. Ognjanov, G. Barać, M. Ljubojević, A. Pranjić, K. Dugalić, S. Ercişli, Turk J Biol, 39, 575-581 (2015). https://doi.org/10.3906/biy-1412-85

4. A. Asănică, V. Tudor, C. Plopa, M. Sumedrea, A. Peticilă, R. Teodorescu, V. Tudor, Agriculture and Agricultural Science Procedia, 10, 172-177 (2016). https://doi.org/10.1016/j.aaspro.2016.09.049

5. J. Dulić, V. Ognjanov, S. Ercisli, M. Miodragović, G. Barać, M. Ljubojevicć, D. Dorić, Erwerbs-Obstbau, 58, 113-118 (2016) https://doi.org/10.1007/s10341-016-0265-y

6. A. Standardi, AgroLife Sci. J., 1(1), 48-54 (2012). http://www.agrolifejournal.usamv.ro/index.php/scientific-papers/48-livestock-2

7. L. R. Nacheva, V. S. Ivanova, J. BioSci. Biotech., 6(3), 187-190 (2017). https://www.researchgate.net/publication/334561573_Silver_nitrate_and_chlorhexidine _gluconate_-_effective_surface_sterilization_agents_in_disinfection_procedures_ at_the_initiation_of_woody_shoot_tip_and_embryo_culture

8. I. Mihaljević, K. Dugalić, V. Tomaš, M. Viljevac, A. Pranjić, Z. Čmelik, B. Puškar, Z. Jurković, J. Agric. Sci., 58(2), 117-126 (2013). https://doi.org/10.2298/JAS1302117M

9. A. Imani, M. Rasouli, R. Tavakoli, E. Zarifi, R. Fatahi, G. Barba-Espín, P. MartínezGómez, Seed Sci. Technol., 39(1), 204-207 (2011). http://doi.org/10.15258/sst.2011.39.1.18

10. K. Martin, J. Sauerborn, Agroecology (Springer, Dordrecht, 49-102, 2013). https://doi.org/10.1007/978-94-007-5917-6_3

11. S. Tariku, T. Lakew, M. Bitew, M. Asfaw, Net J. Agr. Sci., 1(1), 10-16 (2013). https://pdfs.semanticscholar.org/89c8/919377532ea8ae34f91a7d1a3047934c75a9.pdf? $\mathrm{ga}=2.163453180 .1394807965 .1596800063-447193374.1595330244$

12. J. Sochor, P. Babula, V. Adam, B. Krska, R. Kizek, Viruses, 4(11), 2853-2901 (2012). https://doi.org/10.3390/v4112853

13. S. Mariette, M. Tavaud, U. Arunyawat, G. Capdeville, M. Millan, F. Salin, BMC Genetics, 11, 77 (2010). https://doi.org/10.1186/1471-2156-11-77

14. T. A. Natalchuk, T. V. Medvedeva, Ya. S. Zapolskyi, O. B. Barban, Plant varieties studying and protection 16(1), 97-102 (2020). https://doi.org/10.21498/25181017.16.1.2020.201353

15. S. Correia, R. Schouten, A. Silva, B. Gonçalves, Front. Plant Sci., 8, 2166 (2017). https://doi.org/10.3389/fpls.2017.02166 